\title{
A POLYNOMIAL IDENTITY AND ITS APPLICATION TO INVERSE SPECTRAL PROBLEMS IN STIELTJES STRINGS
}

\author{
C. K. Law, V. Pivovarchik And W. C. Wang
}

Abstract. The equation $\Phi=P_{1} Q_{2}+P_{2} Q_{1}$ is studied where $\Phi, Q_{1}, Q_{2}$ are known real polynomials while $P_{1}$ and $P_{2}$ are unknown polynomials. Condition are obtained for the solution $\left(P_{1}, P_{2}\right)$ to exist and to be such that $P_{1}^{-1} Q_{1}$ and $P_{2}^{-1} Q_{2}$ are Stieltjes functions. This result is used to prove the existence of a tree with two complementary subtrees of Stieltjes strings such that the spectrum of the Neumann boundary value problem on the tree is exactly the set of zeros of $\Phi$ and the spectra of Dirichlet problems on the subtrees are the sets of zeros of $Q_{1}$ and $Q_{2}$. This result is generalized to the equation $\Phi=\sum_{i=1}^{q}\left[P_{i} \prod_{j \neq i} Q_{j}\right]$, which is then applied to solve the inverse several spectra problem for trees of Stieltjes strings.

Mathematics subject classification (2010): 34K29, 34K10, 39A70.

Keywords and phrases: Stieltjes function, subtree, Lagrange polynomial, continued fraction, Dirichlet conditions, Neumann conditions.

\section{REFERENCES}

[1] F. V. AtKinson, Discrete and Continuous Boundary Problems, Academic Press, New York, 1964.

[2] O. BOYKO AND V. PIVOVARCHIK, Inverse spectral problem for a star graph of Stieltjes strings, Methods Funct. Anal. Topology 14, 2 (2008), 159-167.

[3] O. BOYKo AND V. PIVOVARCHIK, The inverse three-spectral problem for a Stieltjes string and the inverse problem with one-dimensional damping, Inverse Problems 24, 1 (2008), 015019, 13 pp.

[4] W. CAUER, Die Verwirklichung von Wechselstromwiderständen vorgeschriebenuer Frequenzabhängigkeit, Arch. für Electrotech. 17, 4 (1926), 355-388.

[5] S. Cox, M. EMBREE AND J. HoKAnson, One can hear the composition of a string: experiments with an inverse eigenvalue problem, SIAM Review 54, 1 (2012), 157-178.

[6] M. DRIGNEI, Uniqueness of solutions to inverse Sturm-Liouville problems with $L_{2}(0, a)$ potentials using three spectra, Adv. Appl. Math. 42, 4 (2009), 471-482.

[7] A. DUARTE, Construction of analytic matrices from spectral data, Linear Algebra and Its Applications 113 (1989), 173-182.

[8] A. F. FiLimonov And A. D. MyshKis, On properties of large wave effect in classical problem bead string vibration, J. Difference Equations and Applications 10, 13-15 (2004), 1171-1175.

[9] F. R. Gantmakher And M. G. Krein, Oscillating Matrices and Kernels and Vibrations of Mechanical Systems (Russian), GITTL, Moscow-Leningrad, 1950. German translation: Akademie Verlag, Berlin, 1960.

[10] F. GeSZTESY AND B. SimON, On the determination of a potential from three spectra, in: Advances in Mathematical Sciences, V. Buslaev and M. Solomyak, eds., Amer. Math. Soc. Transl. (2) 189 (1999), $85-92$.

[11] R. O. HRyniv AND YA. V. Mykytyuk, Inverse spectral problems for Sturm-Liouville operators with singular potentials. Part III: Reconstruction by three spectra, J. Math. Anal. Appl. 284, 2 (2003), 626-646.

[12] I. S. KAC AND M. G. KREIN, R-functions - analytic functions mapping the upper half-plane into itself, Amer. Math. Soc. Translations Ser. 2103 (1974), 1-18. 
[13] M. G. KREIN, On some new problems of the theory of vibrations of Sturm systems, Prikladnaya Matematika i Mekhanika 16, 5 (1952), 555-568 (Russian).

[14] P. F. Kurchanov, A. D. Myshkis And A. M. Filimonov, Train vibrations and Kronecker's theorem, Prikladnaya Matematika i Mekhanika 55, 6 (1991), 989-995 (Russian).

[15] C. K. LaW AND V. PIvovarchiK, Characteristic functions of quantum graphs, J. Phys. A: Math. Theor. 42 (2009), 035302 (11p).

[16] B. M. LeVITAN AND M. G. GAS YMov, Determination of a differential equation by two of its spectra, Uspekhi Mat. Nauk 19, 2(116) (1964), 3-63 (Russian).

[17] B. M. Levitan, Inverse Sturm-Liouville Problems (Russian), Moscow, Nauka, 1984. English translation: VNU Science Press BV, Utrecht.

[18] V. Marchenko, Sturm-Liouville Operators and Applications (Russian), Naukova Dumka, Kiev, 1977. English translation: Oper. Theory Adv. Appl. 22, Birkhäuser Verlag, Basel, 1986.

[19] P. Nylen, F. Uhlig, Realization of interlacing by tree-patterned matrices, Linear and Nonlinear Algebra 38 (1994), 13-37.

[20] V. Pivovarchik AND H. Woracek, Sums of Nevanlinna functions and differential equations on star-shaped graphs, Operators and Matrices 3, 4 (2009), 451-501.

[21] V. PIVOVARCHIK, Inverse problem for the Sturm-Liouville equation on a simple graph, SIAM J. Math. Anal. 32 (2000), 801-819.

[22] V. PIVOVARCHIK, Inverse problem for the Sturm-Liouville equation on a star-shaped graph, Math. Nachr. 280 13-14 (2007), 1595-1619.

[23] V. PivovarchiK, Existence of a tree of Stieltjes strings corresponding to two given spectra, J. Phys. A: Math. Theor. 42 (2009), 375213 (16 pp).

[24] V. PIVOVARCHIK, An inverse Sturm-Liouville problem by three spectra, Integral Equations and Operator Theory 34, 2 (1999), 234-243. 